\title{
Mechanical Issues of FIREX Target under Cryogenic Environment ${ }^{*}$
}

\author{
Akifumi IWAMOTO, Takeshi FUJIMURA ${ }^{1)}$, Atsushi SUNAHARA ${ }^{2)}$, Hitoshi SAKAGAMI \\ and Takayoshi NORIMATSU ${ }^{1)}$ \\ National Institute for Fusion Science, 322-6 Oroshi-cho, Toki, Gifu 509-5292, Japan \\ ${ }^{1)}$ Institute of Laser Engineering, Osaka University, 2-6 Yamadaoka, Suita, Osaka 565-0871, Japan \\ ${ }^{2)}$ Institute for Laser Technology, 2-6 Yamadaoka, Suita, Osaka 565-0871, Japan
}

(Received 7 December 2010 / Accepted 22 March 2011)

\begin{abstract}
A typical FIREX target is assembled with a $500 \mu \mathrm{m}$ diameter PS shell, a glass fill tube and a gold cone guide. Each part is glued together by an epoxy resin. To date, several assembled targets have been cooled down to cryogenic environment. However, they could not survive a cool down process and appeared to rupture around the glued boundary between the PS shell and cone guide. To reveal a crucial factor of the target destruction, its thermal stress after cool down was calculated using the ANSYS code. A two dimensional axisymmetric calculation model is composed of the PS shell and cone guide glued by an epoxy resin which is covered on by an epoxy fillet. A cool down process from $293 \mathrm{~K}$ to $10 \mathrm{~K}$ was simulated. The calculation showed that the rupture of the shell would start from the PS shell and gold cone guide boundary and the target validity depended on how the epoxy fillet could reinforce the PS shell.
\end{abstract}

(C) 2011 The Japan Society of Plasma Science and Nuclear Fusion Research

Keywords: mechanical issue of FIREX target, PS shell, epoxy gluing, cryogenic environment, thermal contraction, thermal stress, ANSYS code

DOI: $10.1585 /$ pfr.6.2404070

\section{Introduction}

The National Institute for Fusion Science (NIFS) has collaborated with the Institute of Laser Engineering (ILE), Osaka University to develop the cryogenic target for the Fast Ignition Realization EXperiment (FIREX) project. We apply two strategies of the target development based on the following layering methods: foam shell method [1,2] and cone heating technique [3]. In this paper, a PolyStyrene (PS) shell target for the cone heating technique is focused.

A typical FIREX target consists of a PS shell with $500 \mu \mathrm{m}$ in diameter, a gold cone guide and a glass fill tube. Each part is glued together by an epoxy resin for both structural rigidity and gas leak tightness. Liquid fuel is supplied in the shell though the fill tube, and then it is solidified. A thin layer of solid fuel with $\sim 20 \mu \mathrm{m}$ in thickness is required to be formed on the inner surface of the shell. The developing cone guide heating technique would realize the specification of a fuel layer.

To date, for the convenience of optical observation, a dummy target with a $2 \mathrm{~mm}$ diameter PS shell has been utilized to demonstrate the cone heating technique. No gas leakage has been occurred under cryogenic environment. Recently, several $500 \mu \mathrm{m}$ PS shell targets have been ready for the fuel layering demonstration. However, they have

author's e-mail: iwamoto.akifumi@LHD.nifs.ac.jp

*) This article is based on the presentation at the 20th International Toki Conference (ITC20). not survived a cooling down process yet and have been ruptured. Different thermal contractions of the composing materials might result in exceeding a fracture stress during cool down. To discuss on mechanical issues of an assembled FIREX target under cryogenic environment, the thermal contraction and stress are estimated.

\section{Preliminary Cool Down Test of $500 \mu \mathrm{m}$ PS Shell Target}

Cryogenic validity confirmation of a real sized target has been conducted. The target consists of a PS shell, a glass fill tube and a gold cone guide as shown in Fig. 1. The PS shell is $500 \mu \mathrm{m}$ in diameter and $7 \mu \mathrm{m}$ in thickness. The cone guide is hollow with $2 \mathrm{~mm}$ in length, $\sim 10 \mu \mathrm{m}$ thick-

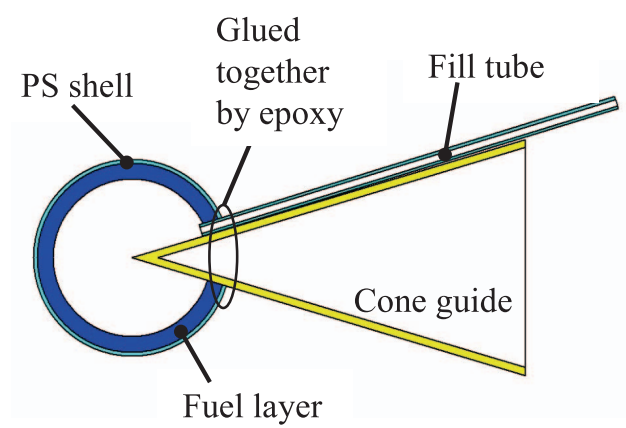

Fig. 1 Schematic of typical FIREX target. 


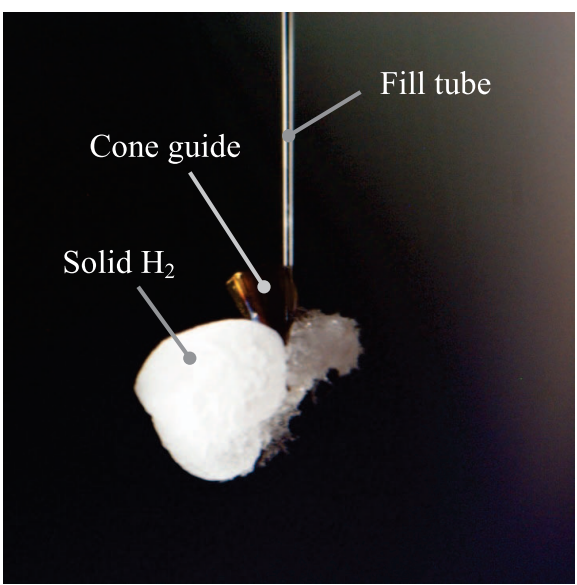

Fig. 2 Example of ruptured target.

ness and $30^{\circ}$ cone angle. Using a laser machining technique [4], a hole was made in the shell, and then a cone guide and a fill tube are inserted into it. A relatively high viscous epoxy resin was utilized to glue the parts together by hand. Therefore, non uniform glue fillet was formed along the PS shell and cone guide boundary. Its leak tightness was confirmed at room temperature using a developed technique [5], and no leakage was detected. A dedicated apparatus was applied for cryogenic experiments [6]. The target was put in a target can and was cooled by ambient gaseous $\mathrm{He}(\mathrm{GHe})$ of $20 \mathrm{~Pa}$. It took $\sim 26$ hours for the target cool down from room temperature to $\sim 9 \mathrm{~K}$. Then ambient GHe temperature was controlled at $13.4 \mathrm{~K}$ where liquefaction temperature was for our fuel layering demonstration. After the temperature became stable, filling gaseous $\mathrm{H}_{2}\left(\mathrm{GH}_{2}\right)$ into the PS shell up to more than $10 \mathrm{kPa}$ of liquefaction pressure was started. However, $\mathrm{H}_{2}$ liquefaction could not be observed and the pressure of the target can gradually increased as the $\mathrm{GH}_{2}$ pressurized time duration. It means that $\mathrm{GH}_{2}$ leaked to the target can through cracks somewhere of the target. Figure 2 represents an example of the ruptured target at cryogenic environment. Solid $\mathrm{H}_{2}$ was suddenly observed around the shell and cone boundary at some ten $\mathrm{kPa}$ of $\mathrm{GH}_{2}$ pressurization, and then it was disappeared. This cycle was continued during the $\mathrm{GH}_{2}$ pressurization. Cool down may initiate cracks from mechanically weak points because different thermal contractions of the materials might result in higher thermal stress than a fracture stress.

\section{Calculation Model of FIREX Tar- get}

Figure 3 shows the two dimensional axisymmetric model of the FIREX target which has the same configuration with the ruptured target. The fill tube, however, is disregarded for model simplification. The shell and cone guide are glued by an epoxy which is covered on by an epoxy fillet. Its radius is varied from 5 to $100 \mu \mathrm{m}$ to esti-

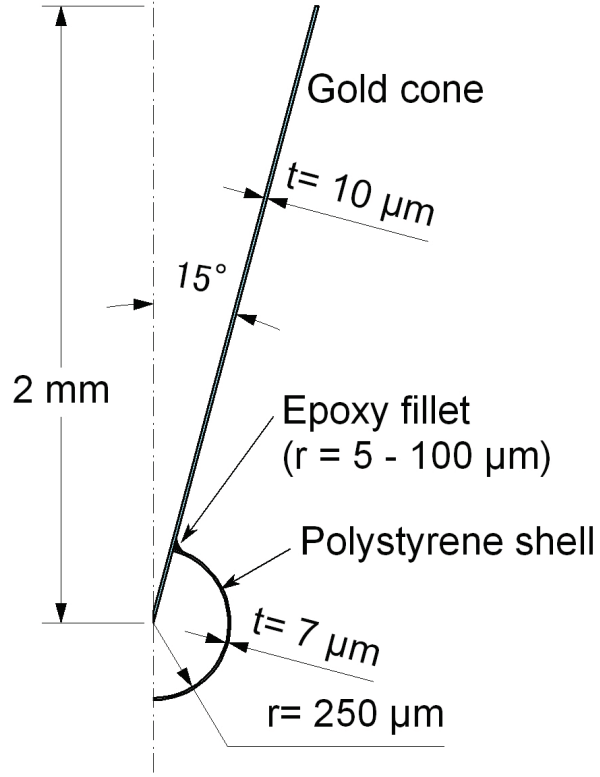

Fig. 3 Calculation model of FIREX target for thermal stress.

Table 1 Material properties for calculation [7-9].

\begin{tabular}{lcc}
\hline & $\begin{array}{c}\text { Thermal contraction } \\
-\Delta L / L_{0}(293-4.2 \mathrm{~K})\end{array}$ & Fracture stress \\
& {$[\%]$} & {$[\mathrm{MPa}]$} \\
\hline PS & 1.5 & $79($ at $4.2 \mathrm{~K})$ \\
Epoxy & 1.1 & $150($ at $4.2 \mathrm{~K})$ \\
Gold & 0.32 & $\sim 200($ at $293 \mathrm{~K})$ \\
\hline
\end{tabular}

mate how the fillet works to keep the target validity. For each component, various thermal contraction coefficients with temperature dependence are considered. Material properties at cryogenic environment are shown in Table 1. Poisson's ratios without temperature dependence are assumed to be $0.34,0.34$ and 0.44 for PS, epoxy and gold, respectively [7]. Cool down from $293 \mathrm{~K}$ to $10 \mathrm{~K}$ is simulated and consequent thermal stress on the target was calculated by the ANSYS code that is a multipurpose finite element analysis software for engineering simulations developed by ANSYS, inc. The point of the cone tip is restricted onto the origin as a constraint.

\section{Calculation of Shrinkage and Ther- mal Stress after Cool Down \\ 4.1 Thermal shrinkage}

Figure 4 represents the deformation of the target after cool down to $10 \mathrm{~K}$. The target has a $5 \mu \mathrm{m}$ epoxy fillet. The PS shell and cone guide shrink the maximum of $\sim 5.6 \mu \mathrm{m}$ and $6.9 \mu \mathrm{m}$, respectively. Before cooling down, the PS shell is a perfect sphere except for the cone guide insertion. Because of the difference of the thermal contraction coefficients between PS and gold, the PS shell is restricted in its uniform thermal shrinkage by the gold cone guide. 


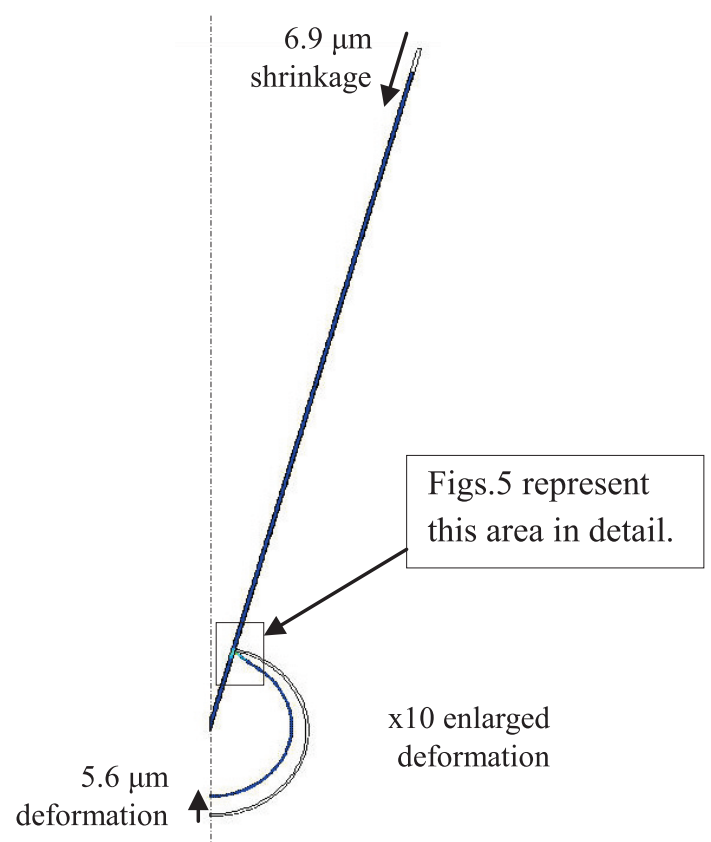

Fig. 4 Shrinkage from cooling down to $10 \mathrm{~K}$.

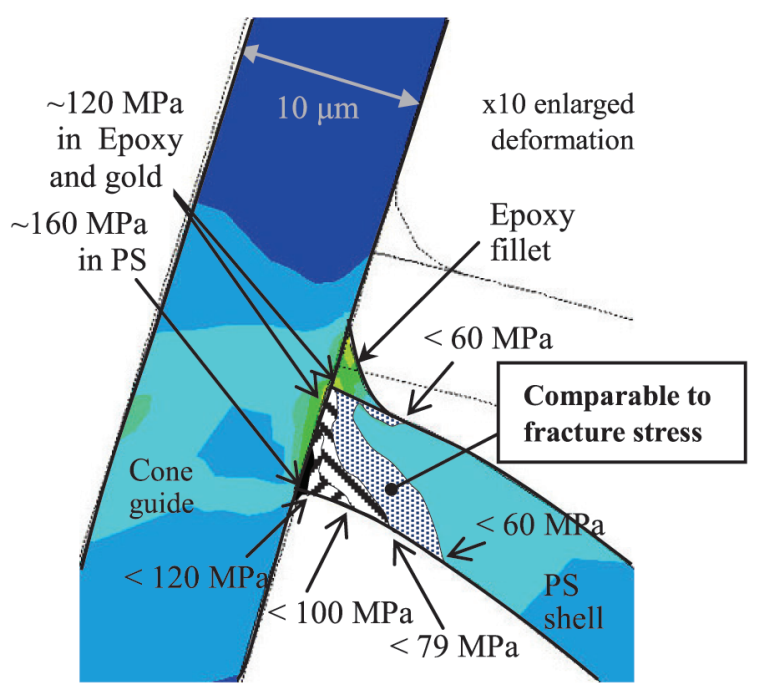

Fig.5 (a) Thermal stress from cooling down to $10 \mathrm{~K}$. Fillet radius $=5 \mu \mathrm{m}$.

Therefore, the shell cannot keep a perfect sphere. According to the brief evaluation of the Legendre polynomial expansion on the PS shell deformation, the mode amplitude on low mode numbers increases after cool down. It might influence uniform fuel compression by the irradiation of implosion lasers to create the high temperature and density plasma required for laser fusion. There is no serious difference of the PS shell and cone guide shrinkages among various fillet radiuses.

\subsection{Thermal stress}

The calculations of total equivalent stress as thermal stress after cooling down with variations of the fillet radius are shown in Figs. 5 (a)-(c). The maximum stress for

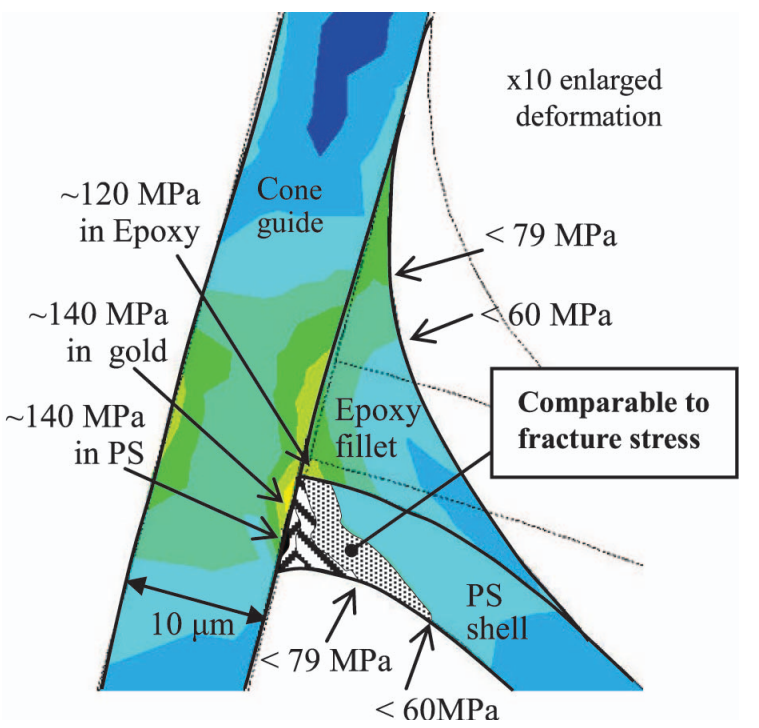

Fig.5 (b) Thermal stress from cooling down to $10 \mathrm{~K}$. Fillet radius $=30 \mu \mathrm{m}$.

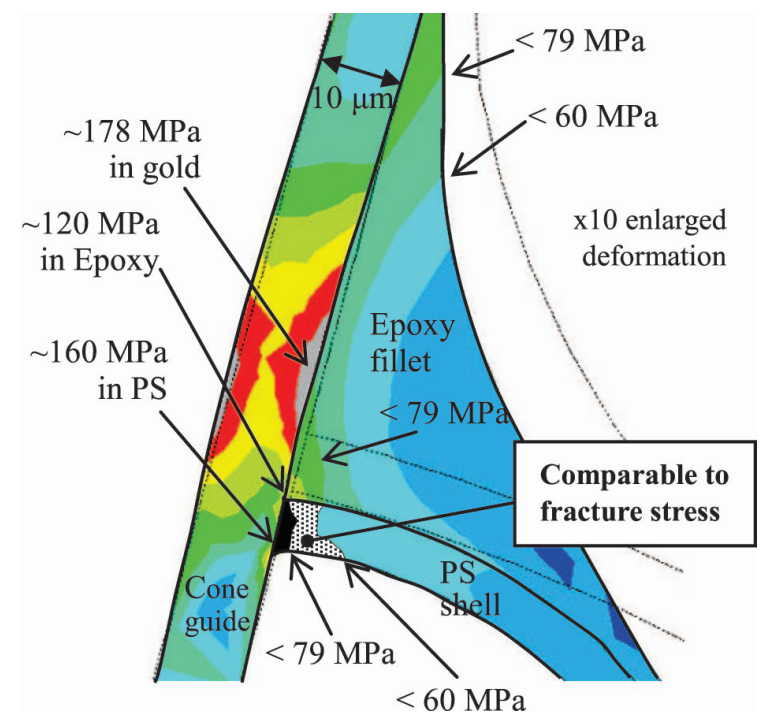

Fig.5 (c) Thermal stress from cooling down to $10 \mathrm{~K}$. Fillet radius $=100 \mu \mathrm{m}$.

each material is evaluated at $140-160 \mathrm{MPa}$ in the PS shell, at $120-178 \mathrm{MPa}$ in the gold cone and at $\sim 120 \mathrm{MPa}$ in the epoxy fillet. The areas exceeding the PS fracture stress are shown in the figures. According to these calculations, along the glued boundary between the cone and the PS shell, the maximum stress in the PS shell is predicted to exceed its fracture stress without fillet radius dependence. A cool down process could destroy the PS shell. For the epoxy fillet, less radius dependence of the maximum stress is expected up to $100 \mu \mathrm{m}$ in radius. On the other hand, the thermal stress in the gold cone is increased as enhancing the epoxy fillet radius but less than its fracture stress. In the range up to a $100 \mu \mathrm{m}$ radius of the fillet, the gold cone can keep its robustness. Finally, it is supposed that the crack 
would initiate from the PS shell and cone guide boundary, and then the epoxy fillet can prevent the rupture of the shell if it has an enough radius for PS shell reinforcement. For example, in the case of a $5 \mu \mathrm{m}$ fillet radius, the fillet does not cover the area under its fracture stress.

\section{Discussions}

The $2 \mathrm{~mm}$ PS shell target was fabricated for the early fuel layering demonstration because of easy optical observation and our unmatured technique. The target has survived for several cool down experiments. Then our fabrication technique has been improved and eventually achieves the successful assembling of the $500 \mu \mathrm{m}$ PS shell target. Several $500 \mu \mathrm{m}$ PS shell targets have been fabricated applying the same technology with the $2 \mathrm{~mm}$ PS shell dummy target. However, cool down tests of the $500 \mu \mathrm{m}$ PS shell targets have not been successful because of gas leakage. It is supposed that the only difference between the two PS shell targets is the epoxy fillet formation. The progressed fabrication technique can realize a minimal fillet, and it must make the target validity degraded according to our calculation. To prevent rupturing of a target, the following countermeasures are proposed: the modification of the epoxy fillet and applying the cone guide material with the similar thermal contraction to that of PS.

For the uniform laser implosion, a perfect hollow spherical fuel layer formed in the shell, except for the cone guide insertion, is demanded. After the fabrication of the target at room temperature, however, cooling down at cryogenic environment results in nonuniform shrinkage of the PS shell. Especially, its deformation around the cone guide would not be neglectable. Solid fuel layer formation will strongly depend on the final appearance of the shrunk PS shell. Altering the material making the cone guide might be a solution. Careful coordination on this issue between the target fabrication and the fast ignition laser fusion physics is essential to decide the final target design.

\section{Conclusions}

The thermal contraction and stress of the $500 \mu \mathrm{m}$ PS shell target after cooling down to $10 \mathrm{~K}$ were calculated using the ANSYS code. The PS shell will shrink nonuniformly. Exceeding the PS fracture stress was predicted around the glued boundary between the PS shell and gold cone guide. Reinforcement by the epoxy fillet with an enough radius may prevent the rupture of the shell. To realize the fast ignition laser fusion, these mechanical issues are not neglectable. Further discussions to decide the final target design are essential.

[1] A. Iwamoto, T. Fujimura, M. Nakai, K. Nagai, T. Norimatsu, R. Maekawa et al., J. Phys.: Conf. Ser. 112, 032067 (2008).

[2] T. Fujimura, A. Iwamoto, M. Nakai, T. Norimatsu, K. Nagai, H. Yang et al., J. Phys.: Conf. Ser. 112, 032066 (2008).

[3] A. Iwamoto, T. Fujimura, M. Nakai, T. Norimatsu, H. Azechi, R. Maekawa et al., J. Phys.: Conf. Ser. 244, 012027 (2010).

[4] T. Fujimura, M. Nakai, A. Iwamoto, N. Hayashi, M. Tanabe, Y. Izawa et al., J. Phys.: Conf. Ser. 244, 032038 (2010).

[5] T. Fujimura, M. Nakai, A. Iwamoto, K. Nagai, H. Homma, K. Tanabe et al., Plasma Fusion Res. 4, S1010 (2009).

[6] A. Iwamoto, R. Maekawa, T. Mito, M. Okamoto, O. Motojima, S. Sugito et al., Fusion Eng. Des. 81, 1647 (2006).

[7] Edited by National Astronomical Observatory, Rika nenpyo: Chronological Scientific Tables 1995 (Maruzen Co. Ltd).

[8] Edited by J.G. Weisend, Handbook of Cryogenic Engineering (Taylor \& Francis).

[9] Cryocomp version 3.06, Cryodata Inc. 